Article

\title{
Experimental Investigation of Centrifugal Flow in Rotor-Stator Cavities at High Reynolds Numbers $>10^{8+}$
}

\author{
Tilman Raphael Schröder *(D), Sebastian Schuster (D) and Dieter Brillert (D)
}

check for updates

Citation: Schröder, T.R.; Schuster, S.; Brillert, D. Experimental Investigation of Centrifugal Flow in Rotor-Stator Cavities at High Reynolds Numbers $>10^{8}$. Int. J. Turbomach. Propuls. Power 2021, 6, 13. https://doi.org/10.3390/ijtpp6020013

Academic Editor: Gerard Bois

Received: 4 May 2021

Accepted: 17 May 2021

Published: 26 May 2021

Publisher's Note: MDPI stays neutral with regard to jurisdictional claims in published maps and institutional affiliations.

Copyright: () 2021 by the authors. Licensee MDPI, Basel, Switzerland. This article is an open access article distributed under the terms and conditions of the Creative Commons Attribution (CC BY-NC-ND) license (https://creativecommons.org/ licenses/by-nc-nd/4.0/)
Chair of Turbomachinery, University of Duisburg-Essen, 47057 Duisburg, Germany; s.schuster@uni-due.de (S.S.); dieter.brillert@uni-due.de (D.B.)

* Correspondence: tilman.schroeder@uni-due.de

+ This manuscript is an extended version of our meeting paper published in the Proceedings of the 14th European Turbomachinery Conference, Gdansk, Poland, 12-16 April 2021.

\begin{abstract}
The designers of radial turbomachinery need detailed information on the impact of the side chamber flow on axial thrust and torque. A previous paper investigated centripetal flow through narrow rotor-stator cavities and compared axial thrust, rotor torque and radial pressure distribution to the case without through-flow. Consequently, this paper extends the investigated range to centrifugal through-flow as it may occur in the hub side chamber of radial turbomachinery. The chosen operating conditions are representative of high-pressure centrifugal compressors used in, for example, carbon capture and storage applications as well as hydrogen compression. To date, only the Reynolds number range up to $R e=2 \cdot 10^{7}$ has been investigated for centrifugal through-flow. This paper extends the range to Reynolds numbers of $R e=2 \cdot 10^{8}$ and reports results of experimental and numerical investigations. It focuses on the radial pressure distribution in the rotor-stator cavity and shows the influence of the Reynolds number, cavity width and centrifugal mass flow rate. It therefore extends the range of available valid data that can be used to design radial turbomachinery. Additionally, this analysis compares the results to data and models from scientific literature, showing that in the higher Reynolds number range, a new correlation is required. Finally, the analysis of velocity profiles and wall shear delineates the switch from purely radial outflow in the cavity to outflow on the rotor and inflow on the stator at high Reynolds numbers in comparison to the results reported by others for Reynolds numbers up to $R e=2 \cdot 10^{7}$.
\end{abstract}

Keywords: radial compressor side chamber; rotor-stator cavity; centrifugal through-flow; axial thrust; radial pressure distribution; Batchelor flow

Most quantities in this paper are dimensionless. The others have a tilde typeset above them: absolute pressure in, e.g., $\mathrm{Pa}$, is denoted by $\widetilde{p}$, its reference value is given by $\widetilde{p}_{\text {ref }}$ and its dimensionless variant by $p$. Quantities averaged over the axial cavity width are written with an overline: $\bar{K}$ is the averaged fluid rotation factor. Vectors are written in boldface.

\section{Introduction}

During the design phase of radial turbomachinery, axial thrust on the rotor must be known with sufficient precision to select axial bearings and ensure reliable operation. Additionally, disc friction torque is needed to estimate machine performance. In the chambers between impeller and casing, rotor-stator cavity flow develops which significantly influences both axial thrust and disc friction. In a previous paper, the authors showed the influence of centripetal through-flow on rotor-stator cavity flow at high Reynolds numbers up to $R e=2 \cdot 10^{8}$. Here, measurements and simulation results of the same test rig are presented, with centrifugal through-flow as it can occur in the side chamber on the hub side of a multistage radial compressor. Only a few experimental investigations of rotor-stator cavity flow with centrifugal through-flow have been published and most focus is on relatively wide cavities. This paper focuses on flow at high Reynolds numbers and small cavity widths. 
Daily and Nece [1] investigated turbulent rotor-stator cavity flow in a test rig able to reach Reynolds numbers up to $R e=1 \cdot 10^{7}$. They discovered that the flow exhibits either separated or merged boundary layers depending on the Reynolds number and cavity width. The flow with separated boundary layers has a rotating core in between them and is often called a Batchelor type flow.

Daily et al. [2] recorded measurements in a rotor-stator cavity test rig filled with either air or water. The test rig superposed centrifugal through-flow on the turbulent rotor-stator cavity flow to investigate its effects on the radial distribution of pressure and the torque needed to drive the disc. This test rig was able to reach Reynolds numbers up to $R e=8 \cdot 10^{6}$ and operated at small and large axial clearances to analyse the flow structures with merged and separated boundary layers. Using the $\frac{1}{7}$ power law for boundary layer velocity profiles and the empirical Blasius relation for shear stress on surfaces, they developed an approximate model for the radial pressure distribution and disc torque increase caused by centrifugal through-flow for the case of large cavity widths. This paper compares their model predictions with measurements recorded in the authors' test rig.

Radtke and Ziemann [3] investigate rotor-stator cavity flow in an air-filled rest rig that operates in the Reynolds number range of $2.1 \cdot 10^{6} \leq \operatorname{Re} \leq 3.0 \cdot 10^{7}$. In their test rig, they analyse the influence of the axial gap width, Reynolds number, centrifugal and centripetal through flow on the velocity distributions, disc torque and pressure distribution in the cavity. This study compares their measurement results to those recorded in the authors' test rig.

Poncet et al. [4] presented experimental and simulation results of a rotor-stator cavity test rig with and without centrifugal through-flow. The test rig reached Reynolds numbers up to $R e=4.15 \cdot 10^{6}$ and operated with water, and the flow was therefore considered incompressible. They measured the radial pressure distribution and fluid velocities in the radial and circumferential direction. A laser Doppler anemometer recorded velocities and delivered the Reynolds stress tensor components in radial, circumferential and combined directions. For their simulations, they validated their own differential Reynolds stress turbulence model that was structurally similar to the SSG model implemented in ANSYS $\mathrm{CFX}^{\mathrm{TM}}$ software. They found that their turbulence model correctly predicted the flow velocities in radial and circumferential directions, but the cavity width slightly influenced that of the radial pressure gradient. This paper compares their measurements and simulation results to those presented here.

\section{Test Rig}

A schematic of the rotor-stator cavity test rig is given in Figure 1. It consists of a disc-shaped rotor which is pivoted inside a cylindrical stator and rotates with angular velocity $\widetilde{\Omega}$. All lengths are given relative to the disc's outer radius $\widetilde{r}_{\text {ref }}$ which is therefore used as the reference length. The disc has a hub radius of $r_{\text {hub }}=0.225$ and a width of $G_{\text {disc }}=0.0625$. It divides the cavity into front and back cavities. The front cavity width can be adjusted to values of $G=0.0125,0.025$ and 0.0375 . The relative radial clearance between disc and casing is $t_{\text {tip }}=0.0125$. Through-flow of mass flow rate $\dot{m}$ enters the front cavity through an annular gap with an outer radius of $r_{\text {gap }}=0.3$. Guide vanes are installed there which ensure a non-swirling axial inflow. The through-flow outlet is located at the shroud in the front cavity at radius $r_{\text {shroud }}=1.0125$ and with a width of $d=3.75 \cdot 10^{-3}$. The test rig operates with carbon dioxide at high pressure. 


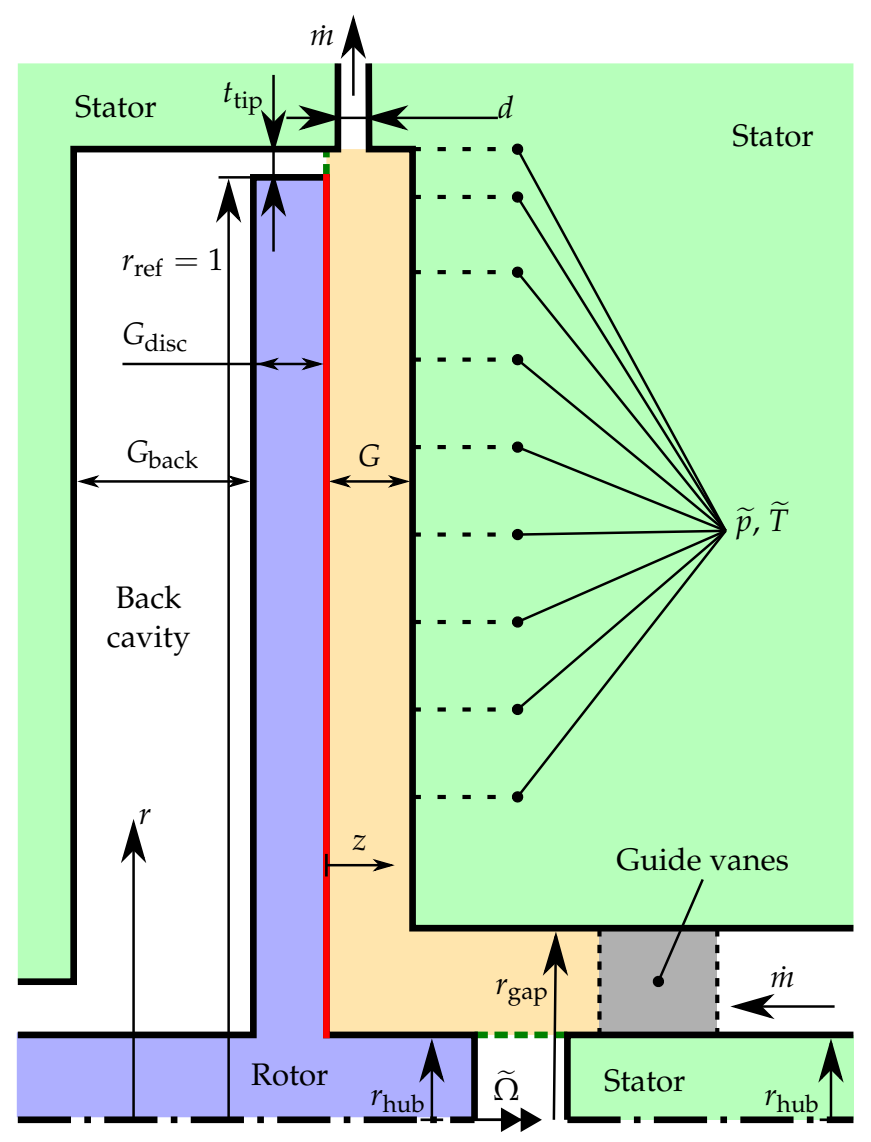

Figure 1. Schematic of the rotor-stator cavity test rig.

Fluid pressure and temperature are measured at the radial positions of $0.42,0.5$, $0.58,0.66,0.74,0.82,0.9,0.97$ and 1.0125 . Afterwards, density and kinematic viscosity are calculated from these measurement results. The radial distribution of these four quantities is then approximated by splines. The absolute values $\widetilde{p}_{\text {ref }}, \widetilde{T}_{\text {ref }}, \widetilde{\rho}_{\text {ref }}$ and $\widetilde{v}_{\text {ref }}$ at a radius of $r_{\text {ref }}=1$ are used as the reference quantities of pressure, temperature, density and kinematic viscosity, respectively. This analysis uses the speed $\widetilde{\Omega} \widetilde{r}_{\text {ref }}$ at the disc's outer periphery as the reference velocity $\widetilde{u}_{\text {ref }}$. Then, the Reynolds number is given by $R e=\frac{\widetilde{r}_{\text {ref }} \widetilde{v}_{\text {ref }}}{\widetilde{v}_{\text {ref }}}=\frac{\widetilde{\Omega} \widetilde{r}_{\text {ref }}^{2}}{\widetilde{v}_{\text {ref }}}$. The dimensionless distributions of pressure, density and velocity are given by $p=\frac{\widetilde{p}-\tilde{p}_{\text {ref }}}{\widetilde{p}_{\text {ref }} \tilde{u}_{\text {ref }}^{2}}$, $\rho=\frac{\widetilde{\rho}}{\tilde{\rho}_{\text {ref }}}$ and $u_{i}=\frac{\widetilde{u}_{i}}{\tilde{u}_{\text {ref }}}$, respectively, with $i=r, \varphi, z$. The dimensionless through-flow mass flow rate $\dot{m}$ is given by $\dot{m}=\frac{\widetilde{m}_{2}}{\widetilde{\rho}_{\text {ref }} \widetilde{u}_{\text {ref }} \widetilde{r}_{\text {ref }}^{2}}$. It can be expressed as the average radial flow velocity $\bar{u}_{r}(r)=\frac{\dot{m}}{2 \pi \rho r G}$ at the disc's outer radius. The test rig is more precisely described in [5].

The geometry of the test rig by Poncet et al. [4] is comparable to the test rig shown in Figure 1. However, Radtke and Ziemann [3] used a different geometry for their investigations of rotor-stator cavity flow with centrifugal through-flow: Their shroud was at a radius of 1.125 compared to 1.0125 in the test rig shown in Figure 1. Additionally, their hub and annular gap outer radii were $r_{\text {hub }}=0.2$ and $r_{\text {gap }}=0.2625$, respectively.

\section{CFD Setup}

The domain investigated using CFD is the orange shaded area shown in Figure 1. The rotor-stator cavity and annular gap around the shaft and stationary hub which ends at the guide vanes comprise the CFD domain. A free slip wall boundary condition separates the rotor-stator cavity from the radial gap between the disc's outer cylindrical surface and the casing. This gap is not part of the CFD domain and the figure shows the boundary 
condition as a dashed dark green line. The same choice is made at the gap between the rotating and stationary parts of the hub. The walls are hydraulically smooth and without slip. The surfaces are analysed with an optical roughness measurement method. It confirmed that the disc's surface is smooth, no roughness structures are detected. The stator's surface roughness is small in the circumferential direction and about $R_{z}=10 \mu \mathrm{m}$ in the radial direction. With centrifugal through-flow, the inlet boundary condition is located at the guide vanes' outlet and imposes the mass flow rate, flow direction and turbulence intensity of $5 \%$. The outlet boundary condition at the shroud enforces the static pressure magnitude there and the outflow direction is a result of the simulation. In the case of a closed cavity without through-flow, the outlet is closed and modelled as a no slip wall while the inlet at the guide vanes is a free slip wall. The fluid density is modelled using the Soave-Redlich-Kwong model. Of all the models available in the CFD software used (ANSYS CFX solver version 19.2), its results are closest to the carbon dioxide data published by Span and Wagner [6] at the pressure and temperature levels occurring in the test rig: Its deviation is, on average, $0.1 \%$, while the second-best model, which is that by Peng and Robinson, deviates on average by $0.3 \%$. The simulation uses the interacting sphere kinetic theory model to calculate the fluid viscosity. The test rig does not measure the wall temperature or heat flux. Thus, boundary conditions for the equation for energy conservation are not available. Instead, the temperature distribution measured in the test rig is imposed. Poncet et al. [4] validated a modified Reynolds stress turbulence model for rotor-stator cavity flows which was based on the work by Elena and Schiestel [7]. It accounted for the implicit effects of rotation on turbulence. In this paper, the SSG Reynolds stress turbulence model is used. Of all Reynolds stress models available in ANSYS CFX 19.2, it is the most similar to that validated by Poncet et al. [4] but it is not sensitised to rotation. A $0.25^{\circ}$ slice of the rotor-stator geometry is used for the simulations and the results are averaged in the circumferential direction. All simulations are steady state. A purely hexahedral mesh, refined at the walls, is used. The dimensionless distance $y^{+}$of the first node away from the wall is smaller than 10 . The turbulence model's wall function is limited to dimensionless wall distances of $y^{+} \geq 11.06$ ([8]), thus a further increase in the near-wall mesh resolution is not required. The solver automatically blends between second and fourth order accuracy of the Rhie-Chow pressure dissipation algorithm. Likewise, the solver's automatic blending between first and second order accurate advection schemes is used. The good agreement of calculated and measured radial pressure distributions validates the CFD setup.

\section{Fluid Rotation Factor}

The fluid rotation factor $K(r, z)=u_{\varphi}(r, z) / r$ is the ratio of the fluid's circumferential velocity to that of the disc at the same radius. In the case of separated boundary layer flow, it refers to the core's rotation. With small cavity widths and merged boundary layers, it is reasonable to use the fluid rotation factor averaged over the axial gap width: $\bar{K}(r)=\frac{1}{G} \int_{0}^{G} K(r, z) \mathrm{d} z$.

To establish a connection between the fluid rotation factor, through-flow mass flow rate and radial distribution of pressure and density, it is assumed that the approximations $\overline{\rho u_{r}^{2}} \approx \bar{\rho}{\overline{u_{r}}}^{2}$ and $\overline{\rho u_{\varphi}^{2}} \approx \bar{\rho}{\overline{u_{\varphi}}}^{2}$ hold. Then, the radial pressure gradient is given by

$$
\frac{\partial \bar{p}}{\partial r} \approx\left[\frac{\bar{\rho}}{r}-\frac{\partial \bar{\rho}}{\partial r}\right] \frac{\dot{m}^{2}}{2 \pi^{2} \bar{\rho}^{2} G^{2} r^{2}}-\bar{\rho} r \bar{K}^{2}
$$

where surface stresses are included in the radial distribution of the averaged fluid rotation factor.

The model by Daily et al. [2] for the radial distribution of the fluid rotation factor is given by

$$
K(r)=K_{0}\left[\dot{m} \operatorname{Re}^{\frac{1}{5}} r^{-\frac{13}{5}} A+1\right]^{-1}
$$


where $K(r)$ and $K_{0}$ are the fluid rotation factors with and without through-flow, respectively, and $A$ is 12.74 . The model assumes separated boundary layer flow with a central core region, velocity profiles in the boundary layers and wall shear stresses that follow the $\frac{1}{7}$ power law and the empirical Blasius law, respectively. Additionally, density is assumed to be constant and radial velocity is taken to be significantly lower than that in the circumferential direction. At the inlet and at the outer disc radius, the fluid rotation factor is assumed to be zero and unchanged by the through-flow, respectively. Daily et al. [2] assumed that $K_{0}=0.5$ holds on all radii. They reported good agreement of (2) with their experimental results at a Reynolds number of $R e=6.9 \cdot 10^{5}$ and axial cavity widths of 0.055 and 0.069 .

Poncet et al. [4] introduced the empirical relation

$$
K(r)=0.032+0.32 \exp \left(-\frac{1}{0.028} \frac{\dot{m}}{2 \pi} \operatorname{Re}^{\frac{1}{5}} r^{-\frac{13}{5}}\right)
$$

for the fluid rotation factor at a cavity width of $G=0.036$. It is validated at a Reynolds number of $\operatorname{Re} \approx 4.2 \cdot 10^{6}$ and assumes constant density, too.

\section{Pressure Distribution}

Figures 2 and 3 show measurements of radial pressure distributions at low Reynolds numbers. The data taken from Radtke and Ziemann [3] were recorded at $R e=4.6 \cdot 10^{6}$, and those from Poncet et al. [4] at $R e=4.2 \cdot 10^{6}$. The test rig operates at $R e=4.6 \cdot 10^{7}$. Data for small and large cavity widths are shown, with the rotor-stator cavity flow exhibiting merged and separated boundary layers, respectively, in the case without through-flow. The radial position of the reference pressure is different for all test rigs, thus the points of zero pressure are individual to each test rig. Therefore, only the pressure distribution's slopes can be compared. All three test rigs show qualitatively similar pressure distributions as the greatest radial gradient is observed without centrifugal through-flow and falling gradients are recorded as through-flow increases. Thus, the authors consider their pressure measurements as reliable.

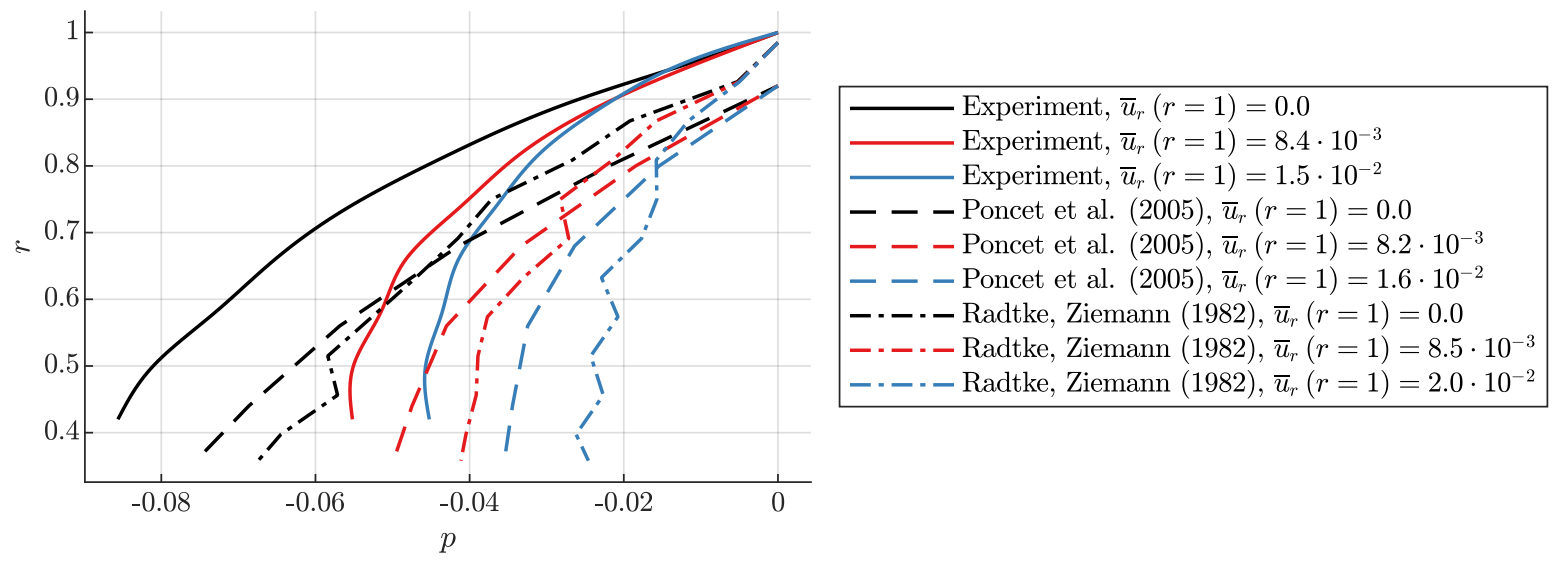

Figure 2. The radial distribution of pressure $p$ with and without centrifugal through-flow at low Reynolds numbers and a small cavity width; the value of $G$ is 0.0125 , only Poncet et al. [4] use $G=0.0120$.

At a small cavity width (see Figure 2), the measurements by Poncet et al. [4] and the authors' test rig exhibit similar radial pressure gradients, although the latter operates at a Reynolds number about ten times higher. Thus, its influence is small compared to that of the through-flow. The results from Radtke and Ziemann [3] show significantly smaller pressure gradients, although the operating points of their test rig were quite close to those of the test rig by Poncet et al. [4]. This effect is attributed to the geometry differences between the test rig by Radtke and Ziemann [3] and that of Poncet et al. [4]. 
At a large cavity width $(0.0360 \leq G \leq 0.0375$, see Figure 3$)$, the test rig shows significantly smaller pressure gradients than that of Poncet et al. [4]. The cavity widths and radial through-flow velocities are similar, only the Reynolds number of the measurements is ten times larger. This shows that at the operating points shown here, the radial pressure gradient decreases as the Reynolds number increases.

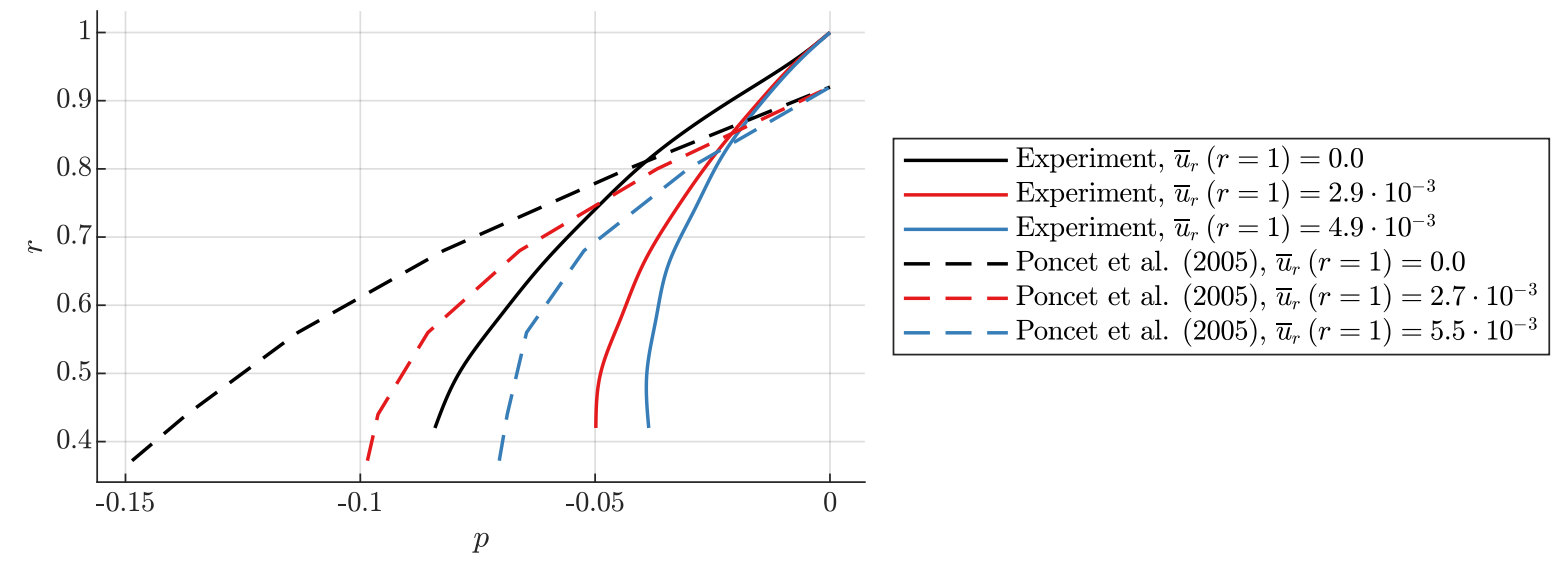

Figure 3. The radial distribution of pressure $p$ with and without centrifugal through-flow at low Reynolds numbers. In the experiment, the cavity width $G$ is 0.0375 . Poncet et al. [4] use $G=0.0360$.

Figure 4 shows the influence of the Reynolds number on the pressure distribution in the case of a small cavity width of $G=0.0125$ : The radial pressure gradient decreases as the Reynolds number rises. The same effect occurs at higher intensity as the centrifugal through-flow increases. Thus, the influence of centrifugal through-flow is significantly higher than that of the Reynolds number in the ranges of $4.6 \cdot 10^{7} \leq \operatorname{Re} \leq 2.5 \cdot 10^{8}$ and $0 \leq \bar{u}_{r}(r=1) \leq 1.4 \cdot 10^{-2}$.
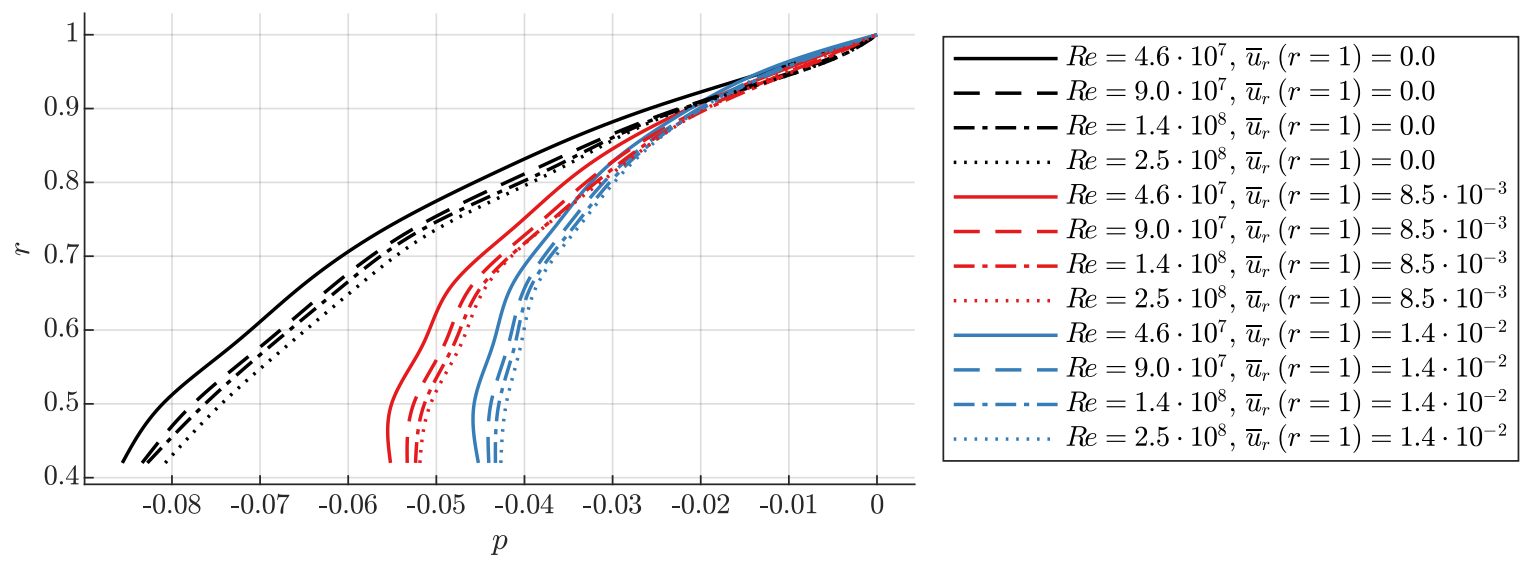

Figure 4. Reynolds number influence on the radial distribution of pressure with and without centrifugal through-flow at the small cavity width. Experimental results.

Figure 5 shows the influence of centrifugal through-flow and cavity width on the radial pressure distribution at a large Reynolds number of $R e=2.5 \cdot 10^{8}$. At all widths, the pressure gradient decreases as the through-flow rises, as expected. Without centrifugal through-flow, the cavity width has little influence on the pressure distribution. In contrast, with centrifugal through-flow, the cavity width exhibits a significant influence: At constant through-flow mass flow rates, a significantly smaller pressure gradient occurs when the cavity width increases from a small value of $G=0.0125$ to a medium value of $G=0.0250$. At the same time, the average radial velocity is halved. In contrast, the pressure gradient does not change as the cavity width increases further to the value of $G=0.0375$ and the 
average radial velocity is $\frac{2}{3}$ of that at $G=0.0250$. This result suggests that at high Reynolds numbers of $R e=2.5 \cdot 10^{8}$ with centrifugal through-flow, the flow structure changes as the cavity width increases from $G=0.0125$ to $G=0.0250$. In contrast, it seems a further increase to $G=0.0375$ does not change the flow structure.

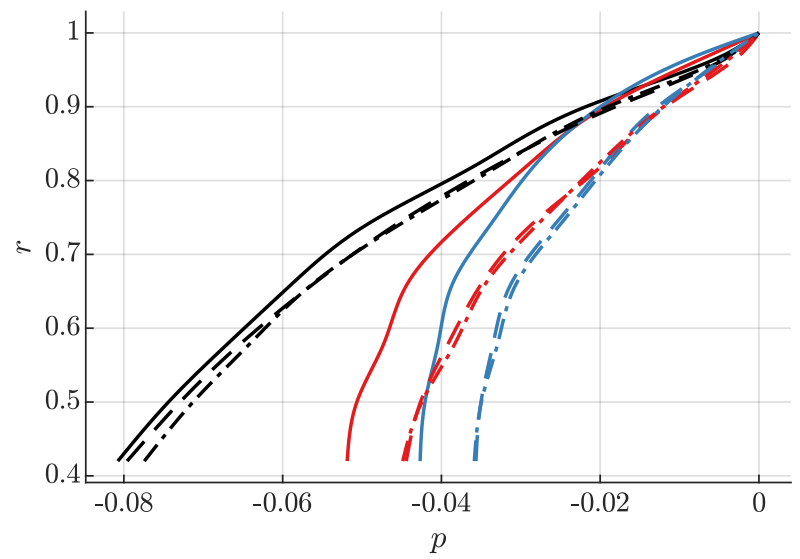

\begin{tabular}{|c|c|}
\hline $\begin{aligned}-G & =0.0125, \bar{u}_{r}(r=1)=0.0, \\
--G=0.0250, \bar{u}_{r}(r=1) & =0.0, \\
-G=0.0375, \bar{u}_{r}(r=1) & =0.0, \\
--G= & 0.0125, \bar{u}_{r}(r=1)=8.5 \cdot 10^{-3}, \\
-G=0.0250, \bar{u}_{r}(r=1) & =4.3 \cdot 10^{-3}, \\
-G & =0.0375, \bar{u}_{r}(r=1)=2.9 \cdot 10^{-3}, \\
-G=0.0125, \bar{u}_{r}(r=1) & =1.4 \cdot 10^{-2},\end{aligned}$ & $\begin{aligned} \dot{m} & =0.0 \\
\dot{m} & =0.0 \\
\dot{m} & =0.0 \\
\dot{m} & =6.6 \cdot 10^{-4} \\
\dot{m} & =6.7 \cdot 10^{-4} \\
\dot{m} & =6.8 \cdot 10^{-4}\end{aligned}$ \\
\hline
\end{tabular}

Figure 5. Cavity width influence on the radial pressure distribution at a high Reynolds number of $\operatorname{Re}=2.5 \cdot 10^{8}$ with and without centrifugal through-flow. Experimental results.

Figure 6 compares the average fluid rotation factor models (2) and (3) by Daily et al. [2] and Poncet et al. [4], respectively, with measurements at a high Reynolds number of $2.5 \cdot 10^{8}$ and the large axial cavity width of 0.0375 . The radial pressure distributions are calculated using (1) together with the measured radial density distributions. The comparison shows that for a closed cavity without through-flow, the models by Daily et al. [2] and Poncet et al. [4] over- and underpredict the radial pressure gradient, respectively. With centrifugal through-flow, both models overestimate the pressure gradient, thus overestimating the influence of the through-flow mass flow rate at high Reynolds numbers.
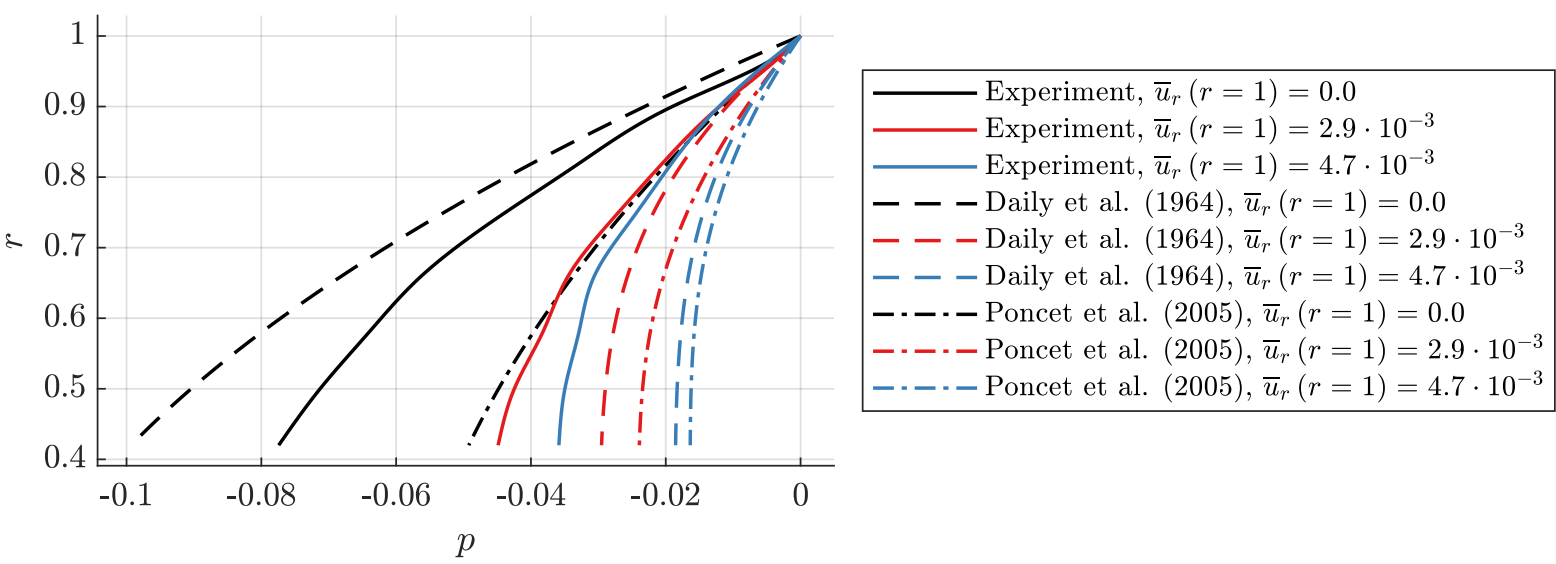

Figure 6. Comparison of the average fluid rotation factor models (2) and (3) by Daily et al. [2] and Poncet et al. [4], respectively, with measurements at the high Reynolds number of $2.5 \cdot 10^{8}$ and an axial cavity width of 0.0375 .

\section{Flow Structure}

Rotor-stator cavity flow without through-flow may exhibit separated boundary layers on the rotor and stator with a core region in between which has zero radial and constant circumferential velocity. This structure is called Batchelor flow. With centrifugal throughflow, Stewartson flow can occur in which the circumferential velocity component forms a boundary layer on the rotor only and approaches zero towards the cavity middle and is zero on the stator. The radial component is then positive at all axial positions. 
The flow at the high Reynolds number of $\operatorname{Re}=2.5 \cdot 10^{8}$, at a high through-flow mass flow rate of $\dot{m}=1.1 \cdot 10^{-3}$ and at all three cavity widths is further investigated using CFD. Figure 7a compares the measured and calculated radial pressure distributions: The cavity width influence is reproduced by the simulations, although CFD results show higher pressure magnitudes than the experiments. Thus, the simulations can be used to investigate the flow structure.

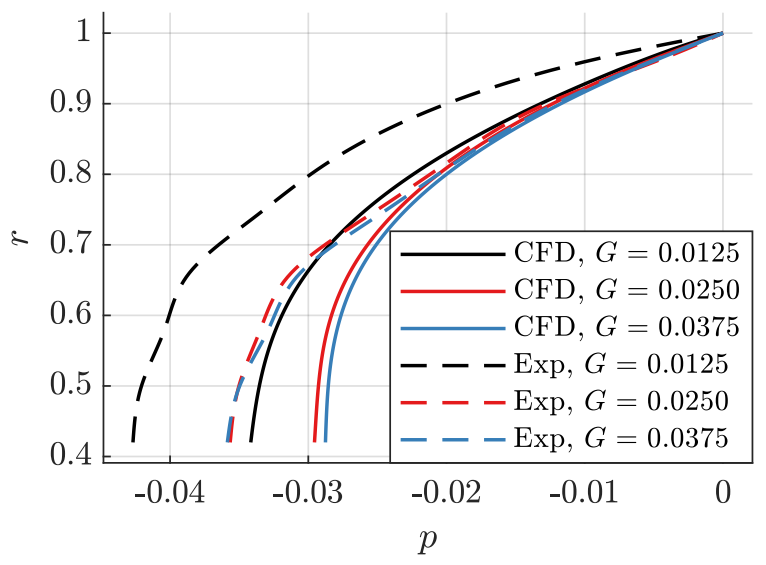

(a) Pressure distribution, CFD versus experiment.

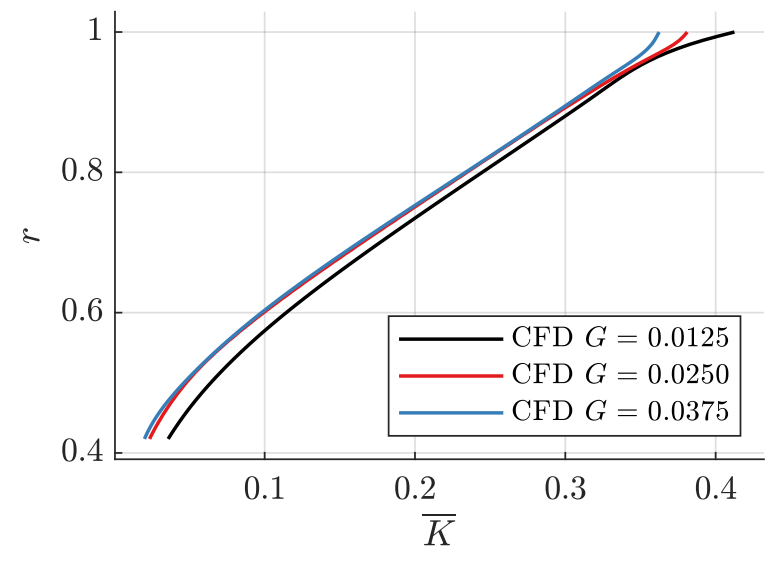

(b) Fluid rotation factor according to CFD.

Figure 7. Radial distribution of pressure and fluid rotation factor at the Reynolds number of $R e=2.5 \cdot 10^{8}$, through-flow mass flow rate of $\dot{m}=1.1 \cdot 10^{-3}$ and at different cavity widths.

The behaviour of the pressure distributions correlates with the average fluid rotation factor $\bar{K}$ (see Figure $7 b$ ): A high fluid rotation factor leads to a higher radial pressure gradient. The circumferential velocity component has, at all radii $r \geq 0.44$ and cavity widths, a stator-side boundary layer and a core region where the fluid rotation factor is constant (see Figure $8 \mathrm{a}$ for the radius of 0.56 ). Thus, the circumferential velocity is of the Batchelor type. At the same radius, the radial velocity component strongly depends on the axial cavity width: The radial velocity component is positive at all axial positions when the cavity width is small, and changes to a profile with a negative radial velocity component at the stator as the axial cavity width increases. This shows that the circumferential and radial velocity profiles develop mostly independent from each other.

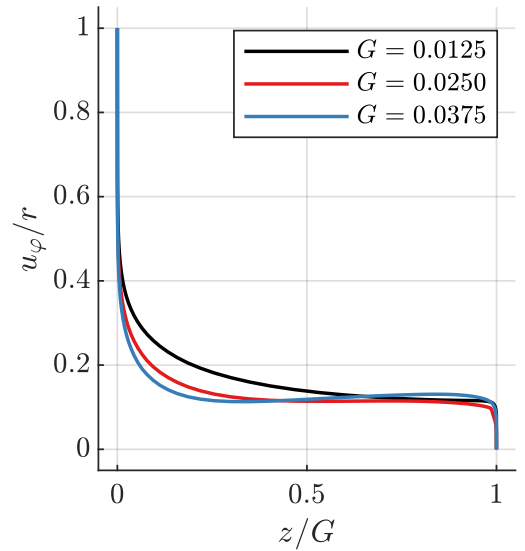

(a) Circumferential velocity.

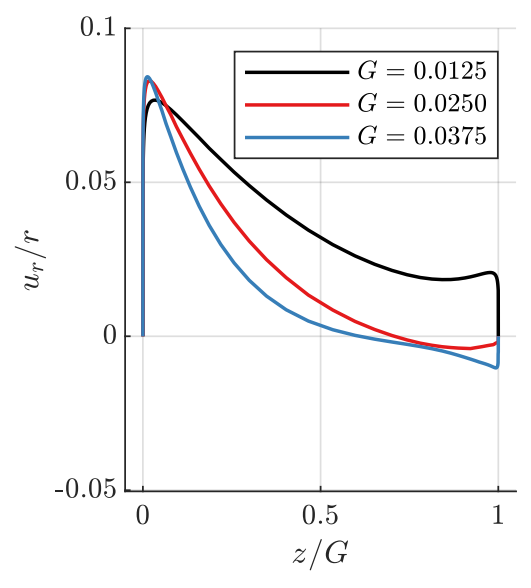

(b) Radial velocity.

Figure 8. Axial profiles of radial and circumferential velocity components relative to the disc speed at the radius of 0.56 and at the Reynolds number of $R e=2.5 \cdot 10^{8}$, through-flow mass flow rate of $\dot{m}=1.1 \cdot 10^{-3}$ and at different cavity widths. Simulation results.

Figure $9 \mathrm{~b}$ shows the transition of the Batchelor type axial profile of the radial velocity component at a radius of 0.92 from a merged boundary layer structure at the small 
cavity width to one with separated boundary layers at the large cavity width. At the latter, all centrifugal through-flow is entrained in the rotor-side boundary layers and a core region exists where the radial velocity component vanishes. Figure 9a shows the circumferential velocity profiles at the same radius and of the same three operating points. The profiles are only slightly influenced by the change in the axial cavity width: The slope $\partial\left[u_{\varphi} / r\right] / \partial[z / G]$ changes its sign from negative to positive as the axial cavity width increases from 0.0125 to 0.0250 . A further increase in the cavity width to 0.0375 does not change the slope significantly. The average fluid rotation factor $\bar{K}$ does not change as the cavity width changes.

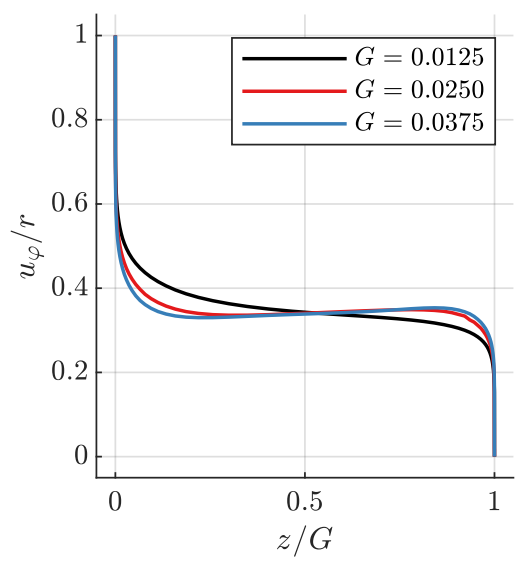

(a) Circumferential velocity.

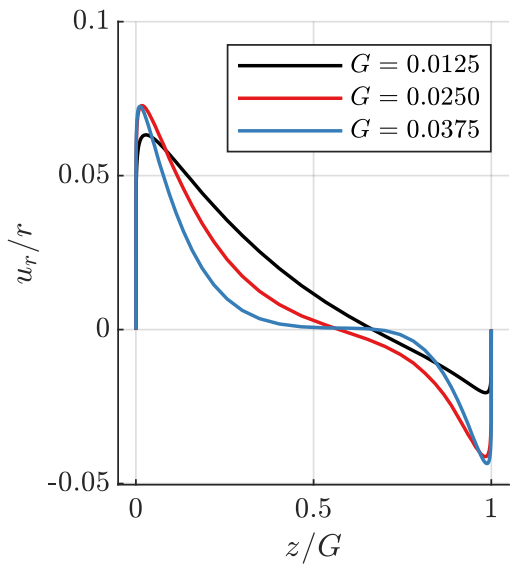

(b) Radial velocity.

Figure 9. Axial profiles of radial and circumferential velocity components relative to the disc speed at the radius of 0.92 and at the Reynolds number of $R e=2.5 \cdot 10^{8}$, through-flow mass flow rate of $\dot{m}=1.1 \cdot 10^{-3}$ and at different cavity widths. Simulation results.

The wall shear stress angle $\gamma$ at the stator shows the switch from purely radial outflow in the cavity to outflow on the rotor and inflow on the stator. It is defined as $\tan (\gamma(z=G))=\frac{-\tau_{\varphi q}(z=G)}{-\tau_{r z}(z=G)}$. The values of $\gamma=0^{\circ}, \gamma=180^{\circ}, \gamma=90^{\circ}$ and $\gamma=270^{\circ}$ denote radial outward, radial inward, positive circumferential and negative circumferential directions, respectively. The radial velocity component on the stator switches from outflow to inflow when the angle $\gamma$ switches from $\gamma(z=G)<270^{\circ}$ to $\gamma(z=G)>270^{\circ}$. The wall shear stress angle at the stator is opposite to the velocity vector close to it, see Figure 10 for examples.

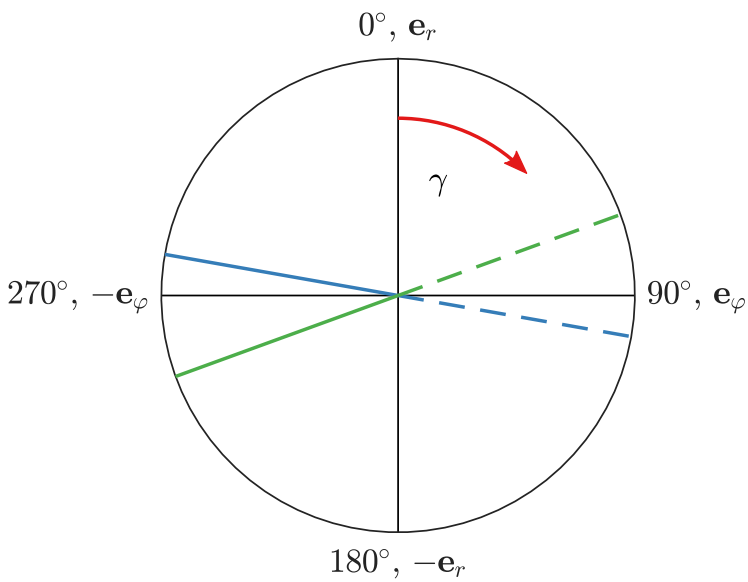

Figure 10. Definition of the wall shear stress angle $\gamma$. 
Figure 11 shows the wall shear stress angle on the stator from the CFD simulations described above. The switch happens at the radii of $r \approx 0.68, r \approx 0.54$ and $r \approx 0.47$ for the cavity widths of $G=0.0125, G=0.0250$ and $G=0.0375$, respectively.

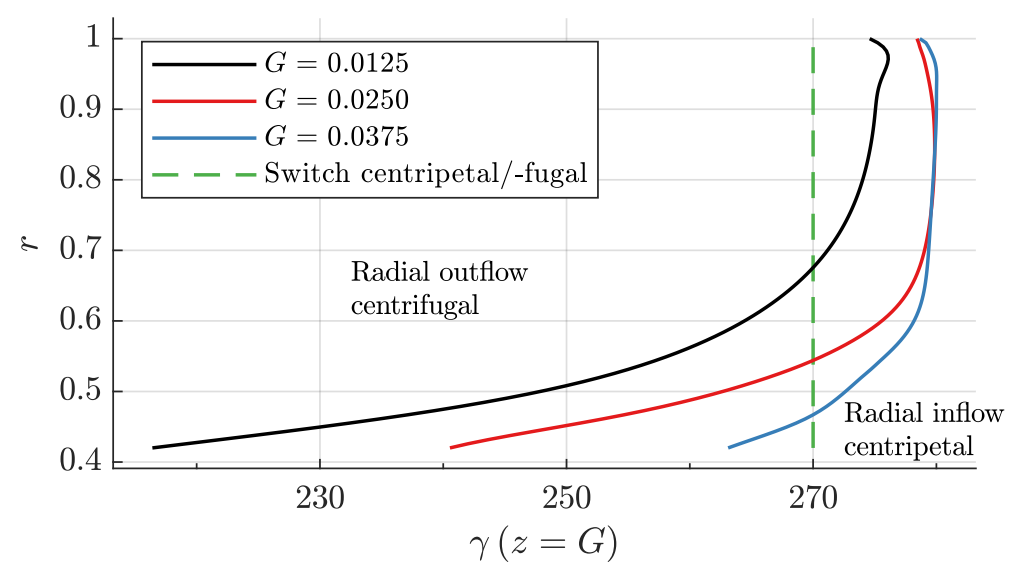

Figure 11. Wall shear stress angle $\gamma$ on the stator $(z=G)$ at the Reynolds number of $\operatorname{Re}=2.5 \cdot 10^{8}$, through-flow mass flow rate of $\dot{m}=1.1 \cdot 10^{-3}$ and at different cavity widths. Simulation results.

\section{Conclusions}

Rotor-stator cavity flow with centrifugal through-flow in the Reynolds numbers range of $4 \cdot 10^{7} \leq R e \leq 2 \cdot 10^{8}$ is studied experimentally and numerically. At all investigated Reynolds numbers and cavity widths, centrifugal through-flow influences the flow and causes significantly lower radial pressure gradients. This influence is substantially larger than that of the Reynolds number according to the experimental data at small cavity widths. However, the cavity width plays an important role: The measurements show that at high Reynolds number and with centrifugal through-flow, the radial distributions of pressure and average fluid rotation factor change significantly when switching the cavity width from $G=0.0125$ to $G=0.0250$, but do not change when increasing it further to $G=0.0375$. Two empirical models for the average fluid rotation factor overestimate the influence of the centrifugal mass flow rate at high Reynolds numbers and the large cavity width of $G=0.0375$, thus leading to radial pressure gradients that are smaller than that measured in the test rig. Using CFD, the flow structure at these operating points is investigated: The wall shear stress angle on the stator shows the switch of the radial velocity component from an exclusively outward flowing profile to that of a two-layer structure with outward and inward fluid flow on the rotor and stator, respectively. The axial profiles of the circumferential velocity component are not significantly influenced by the change in the axial cavity width, showing that both components can change mostly independent of each other.

Author Contributions: Conceptualisation, T.R.S.; Data curation, T.R.S.; Formal analysis, T.R.S.; Funding acquisition, D.B.; Investigation, T.R.S.; Methodology, T.R.S.; Project administration, D.B.; Resources, T.R.S.; Software, T.R.S.; Supervision, S.S.; Validation, T.R.S.; Visualisation, T.R.S.; Writingoriginal draft, T.R.S.; Writing-review and editing, S.S. and D.B. All authors have read and agreed to the published version of the manuscript.

Funding: This research received no external funding.

Institutional Review Board Statement: Not applicable.

Informed Consent Statement: Not applicable.

Data Availability Statement: Not applicable.

Conflicts of Interest: The authors declare no conflict of interest. 


\begin{tabular}{ll}
\multicolumn{2}{l}{ Nomenclature } \\
CFD & Computational fluid dynamics \\
$d$ & Width of outlet at the shroud \\
$e$ & Unit vector \\
$G$ & Cavity width \\
$i$ & An index \\
$K$ & Fluid rotation factor \\
$l$ & Length \\
$\dot{m}$ & Mass flow rate of through-flow \\
$p$ & Pressure \\
$r$ & Radius \\
$R e$ & Reynolds number
\end{tabular}

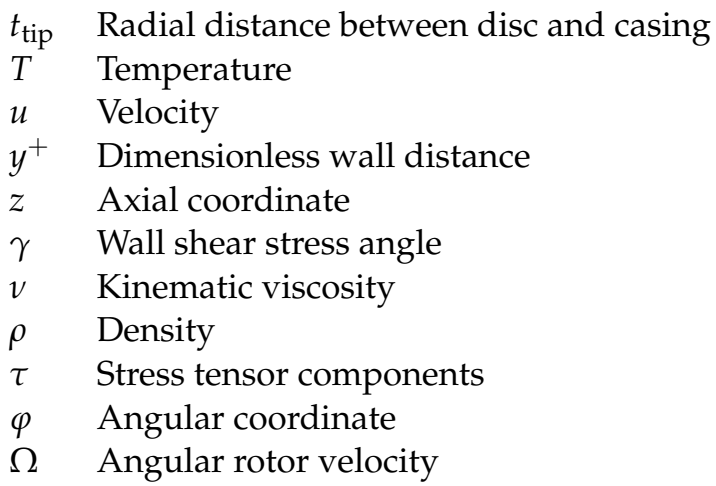

\section{References}

1. Daily, J.W.; Nece, R.E. Chamber Dimension Effects on Induced Flow and Frictional Resistance of Enclosed Rotating Disks. J. Basic Eng. 1960, 82, 217-230. [CrossRef]

2. Daily, J.W.; Ernst, W.D.; Asbedian, V.V. Enclosed Rotating Disks with Superposed Throughflow: Mean Steady and Periodic Unsteady Characteristics of Induced Flow; Hydrodynamics Laboratory Reports; Department of Civil Engineering, Massachusetts Institute of Technology: Cambridge, MA, USA, 1964; Volume 64.

3. Radtke, F.; Ziemann, M. Scheibenreibung-Vorhaben Nr. 213-Experimentelle und Theoretische Untersuchungen des Reibungseinflusses an Rotierenden Scheiben-Abschlussbericht; Technical Report; Institut für Dampf- und Gasturbinen, Rheinisch-Westfälische Technische Hochschule Aachen: Aachen, Germany, 1982.

4. Poncet, S.; Schiestel, R.; Chauve, M.P. Centrifugal Flow in a Rotor-Stator Cavity. J. Fluids Eng. 2005, 127, 787-794. [CrossRef]

5. Schröder, T.R.; Dohmen, H.J.; Brillert, D.; Benra, F.K. Impact of Leakage Inlet Swirl Angle in a Rotor-Stator Cavity on Flow Pattern, Radial Pressure Distribution and Frictional Torque in a Wide Circumferential Reynolds Number Range. Int. J. Turbomach. Propuls. Power 2020, 5, 7. [CrossRef]

6. Span, R.; Wagner, W. A New Equation of State for Carbon Dioxide Covering the Fluid Region from the Triple Point Temperature to $1100 \mathrm{~K}$ at Pressures up to $800 \mathrm{MPa}$. J. Phys. Chem. Ref. Data 1996, 25, 1509-1596. [CrossRef]

7. Elena, L.; Schiestel, R. Turbulence modeling of rotating confined flows. Int. J. Heat Fluid Flow 1996, 17, 283-289. [CrossRef]

8. $\quad$ ANSYS, Inc. CFX 19.2 Theory Guide; ANSYS, Inc.: Canonsburg, PA, USA, 2018. 